\title{
A Study on role of socio demographic status of women in excessive vaginal discharge at teaching hospital
}

\author{
Sharda B Ahmed \\ Associate Professor, Department of Obstetrics and Gynaecology, American International Institute of Medical Sciences, Udaipur, Rajasthan, \\ India \\ *Corresponding Author: Sharda B Ahmed \\ Email: drshardaahmed@gmail.com
}

Received: $18^{\text {th }}$ April, 2019

Accepted: $28^{\text {th }}$ May, 2019

\begin{abstract}
Introduction: The complaint of excessive vaginal discharge is very common in women attending the gynecology out-patient department. It is associated with considerable emotional stress, health seeking associated cost and has a substantial impact on female health and behavior Objective: To find out socio-demographic variables associated with the complaint of vaginal discharge wherein no biomedical cause of leucorrhoea can be detected. Socio-cultural perspectives have an exaggerative effect on the normal vaginal discharge and in absence of pathological diagnosis, they create fear and anxiety in couples. The problem of vaginal discharge can be best understood not only in the biomedical perspective but also in the socio cultural perspective.

Materials and Methods: This is a hospital based study, conducted in out-patient department of obstetrics and gynecology department, AIIMS, Udaipur (Raj). Women with complaint of vaginal discharge without any evident pathological or infectious causes were counted in the study. The women with vaginal discharge which is non-infected, non-blood stained, non-purulent, without pruritus vulvae were interviewed with a pretested questionnaire. Socio-demographic details, personal and menstrual hygiene, obstetric history and contraceptive practices were noted and tabulated.

Conclusion: To mitigate the problem of vaginal discharge, socio-demographic status of women should be taken in consideration. Improvement of literacy rate, economic status and women's empowerment are the key areas of interventions. Proper understanding of this problem helps in initiating appropriate medical education programs and framing health policies.
\end{abstract}

Keywords: Excessive vaginal discharge, Socio-demographics, Somatic symptoms, Leucorrhoea, Pruritus tubectomy.

\section{Introduction}

Excessive vaginal discharge is one of the most common complaints in women attending the obstetrics and gynecology out-patient department. Every fourth woman coming to outdoor is present with agony of excessive vaginal discharge ${ }^{1}$. After investigating, in most of women, causative underlying pathology or infection is detected and hence managed accordingly. But in few women, no cause is evident and in absence of definite diagnosis, it is very difficult to treat the women and to make her symptom free. Even after thorough counseling, unseen fears make them consult one and other gynecologist now and then. Hence excessive vaginal discharge affects the physical, sexual and social activities of women and also creates anxiety in couples.

The vagina is normally kept moist by transudation of fluid through the vaginal wall, which mixes with the exfoliated vaginal cells, aliphatic acid, microorganism and mucous from cervix ${ }^{2}$. The quantity of vaginal \& cervical secretions varies through menstrual cycle, peaking at ovulation, before \& after menstruation, during pregnancy and by sexual excitement. ${ }^{3}$ This physiological excess may be explained on the basis of variation in endogenous hormonal levels, especially estrogen ${ }^{3}$ hence deviation in Hypothalamus-pituitary-ovarian axis of hormones can be causative factor for excessive vaginal discharge. Jeffcoate describes excessive amount of normal vaginal discharge, a running of white substance, as leucorrhoea. ${ }^{4}$ The term 'leucorrhoea' should be restricted to those patients in whom the normal vaginal secretion is increased in amount. It should be non-infective, not purulent, non blood stained, never causes pruritus \& never offensive. ${ }^{3,4}$ It is evident by persistent moistness of vulva or staining of underclothes. The amount and severity of complaint of excessive vaginal discharge depends on power of observation, understanding and hygiene of women and family. In absence of definite diagnosis, patient tends to exaggerate her complaint into something seriously pathological and it causes distressing alarm in family from fear of venereal diseases or cancer. ${ }^{4}$ It may restrict her domestic and occupational work resulting in social and economic implications.

Excessive vaginal discharge is referred as safedpanni, swedpradhar or dhattu in local language in North India. Medically it is labeled as Leucorrhoea. Studies conducted by Vishwanath $\mathrm{S}$ in India and Hawkes in Bangladesh shown that vaginal discharge had laboratory evidence of infection only in $60 \%$ \& $30 \%$ women respectively and in other women no infectious cause was detected. ${ }^{6,7}$ Chaudhary V concluded that increasing age, illiteracy, low socioeconomic status, high parity, induced abortion and place of delivery are all contributory for occurrence of vaginal discharge. ${ }^{8}$ Depression, sexual violence, verbal abuse, low social autonomy and integration have also been found to be associated with leucorrhoea. Psychosocial adversity and vaginal discharge has a strong association shown in study by Patel V in South Asian women. ${ }^{9}$ Mental tension can be a cause of any illness has also been described. There are 
multiple vague somatic symptoms as weakness, leg cramps, backache, giddiness, pain abdomen etc associated with vaginal discharge. Most of women had one or more theories regarding causative origin of discharge as eating of hot food, warmth of some strong medicine or date back to some event as fever, abortion or tubectomy etc. So not only biomedical context is important in managing vaginal discharge but understanding of socio cultural perspective has a significant role.

\section{Materials and Methods}

This is hospital based, cross sectional study conducted in obstetrics and gynecology out-patient department AIIMS Udaipur (Raj). The study period was from Nov 2017 to April 2018. All married women attending the out-patient department with complain of excessive vaginal discharge were examined and investigated to find out any pathological or infectious cause. The patients with symptom of excessive vaginal discharge, which was non-purulent, not blood stained or fowl smelling and not associated with itching were included in the study. No organic pathology was detected in these women and no WBC found in discharge. A pretested questionnaire was used to interview them. There socio-demographic details, menstrual history \& hygiene, detailed obstetrical history, contraceptives used were noted. Details of vaginal discharge, their perception of causes and effect of discharge, any other associated morbidity and any other treatment taken were inquired and documented.

\section{Results}

Out of 3000 women attending gynecology outdoor patient department, 720 patients were having complaint of excessive vaginal discharge, i.e., $24 \%$ prevalence rate. After excluding all organic and infectious causes, 82 patients were left behind, in whom no definite pathological diagnosis could be reached. In these women, discharge was found to be non-infected, non-purulent, not blood stained and not associated with pruritus.

In this study, 59\% women were in age group of 25-34 years, 30\% women were in age group of 35-44 years. Before 25 and after 45 years, discharge was mostly associated with some underlying pathology (Table 1).

Table 1: Age Distribution of Study Population

\begin{tabular}{|c|c|c|}
\hline Age Group (years) & Total Number & Percentage \\
\hline $15-24$ & 07 & $09 \%$ \\
\hline $25-34$ & 48 & $59 \%$ \\
\hline $35-44$ & 25 & $30 \%$ \\
\hline$>45$ & 02 & $02 \%$ \\
\hline
\end{tabular}

Association of excessive vaginal discharge with socio-demographic characters shows $83 \%$ of women were from low socioeconomic group, $87 \%$ women were belong to rural area, $71 \%$ women were illiterate, $61 \%$ women were working either in farms or at construction sites and $26 \%$ respondents were home makers (Table 2).

Table 2: Showing demographic characteristics of study women

\begin{tabular}{|c|c|c|c|}
\hline Characteristic & Classification & No of Women & Percentage \\
\hline Economic Status & Low & 68 & $83 \%$ \\
\cline { 2 - 4 } & High & 14 & $17 \%$ \\
\hline \multirow{2}{*}{ Literacy Status } & Literate & 24 & $29 \%$ \\
\cline { 2 - 4 } & Illiterate & 58 & $71 \%$ \\
\hline \multirow{3}{*}{ Locality } & Rural & 71 & $87 \%$ \\
\cline { 2 - 4 } & Urban & 11 & $13 \%$ \\
\hline Occupational Status & Home-Maker & 21 & $26 \%$ \\
\cline { 2 - 4 } & Farmer & 23 & $28 \%$ \\
\cline { 2 - 4 } & Labourer & 27 & $33 \%$ \\
\cline { 2 - 4 } & Skilled Worker & 11 & $13 \%$ \\
\hline
\end{tabular}

On distribution of women according to their personal and menstrual hygiene, it was found that $70 \%$ women were used to open air defecation, may be because of old habits or scarcity of water. $82 \%$ women were not using the sanitary napkins and their menstrual hygiene was very pitiful. Few women can't afford and few even don't know about sanitary napkins (Table 3 ).

Table 3: Personal \& menstrual hygiene

\begin{tabular}{|c|c|c|c|}
\hline Group & Classification & No of Women & Percentage \\
\hline \multirow{2}{*}{ Type of Toilet } & Open air defecation & 57 & $70 \%$ \\
\cline { 2 - 4 } & Commode & 25 & $30 \%$ \\
\hline \multirow{2}{*}{ Usage of Pads } & Sanitary Pads & 15 & $18 \%$ \\
\cline { 2 - 4 } & No Pads (using cloth) & 67 & $82 \%$ \\
\hline
\end{tabular}


$63 \%$ women were gravida three or more in this study. Home deliveries $(52 \%)$ were more as compared to institutional deliveries (44\%). In this study $65 \%$ women had undergone tubectomy while $28 \%$ women were not using any contraceptives. Interest in using temporary methods of contraception was very low as they had many types of fears (Table 4).

Table 4: Gravida, place of delivery \& contraception

\begin{tabular}{|c|c|c|c|}
\hline Group & Classification & No of Women & Percentage \\
\hline \multirow{4}{*}{ Gravida } & Nullipara & 03 & $04 \%$ \\
\cline { 2 - 4 } & One & 06 & $07 \%$ \\
\cline { 2 - 4 } & Two & 21 & $26 \%$ \\
\cline { 2 - 4 } & Three & 52 & $63 \%$ \\
\hline \multirow{3}{*}{ Place of Delivery } & Home & 43 & $52 \%$ \\
\cline { 2 - 4 } & Institutional & 36 & $44 \%$ \\
\cline { 2 - 4 } & Both & 03 & $04 \%$ \\
\hline Contraception & Nil & 23 & $28 \%$ \\
\cline { 2 - 4 } & Temporary & 06 & $65 \%$ \\
\cline { 2 - 4 } & Permanent & 53 & \\
\hline
\end{tabular}

Most of the women in this study were having some associated symptom, which cannot be explained on the basis of white discharge only. All these symptoms seem to be somatic rather than pathological. Majority of women were reported with associated complaint of weakness in $82 \%$, leg cramps in $73 \%$, backache in $51 \%$, general body aches in $36 \%$ and pain abdomen in $17 \%$. In few women visual problems like diminishing of vision, gastric problems like gas formation, distension of abdomen and infertility were also present along with vaginal discharge. $17 \%$ women had no symptom other than white discharge. There was a mass confusion in women, whether accompanied symptoms were cause or result of excessive vaginal discharge (Table 5).

Table 5: Perceived symptoms associated with vaginal discharge

\begin{tabular}{|c|c|c|}
\hline Perceived Symptom & No. of Women & Percentage \\
\hline Weakness & 68 & $82 \%$ \\
\hline Leg Cramps & 60 & $73 \%$ \\
\hline Backache & 42 & $51 \%$ \\
\hline Bodyache & 30 & $36 \%$ \\
\hline Pain Abdomen & 14 & $17 \%$ \\
\hline Weight Loss & 06 & $07 \%$ \\
\hline Visual Problem & 03 & $03 \%$ \\
\hline Gastric Problems & 07 & $07 \%$ \\
\hline Infertility & 01 & $01 \%$ \\
\hline No Symptoms & 14 & $17 \%$ \\
\hline
\end{tabular}

Women prefixed multiple theories as regard to origin of vaginal discharge. Some date back to some event or happenings as tubectomy, fever, eating of hot food, strong medicine, previous abortion or bad delivery.

Table 6: Perceived causes of excessive vaginal discharge

\begin{tabular}{|c|c|c|}
\hline Perceived Cause & No of Women & Percentage \\
\hline Weakness & 39 & $47 \%$ \\
\hline Excess Body Heat & 19 & $23 \%$ \\
\hline Tubectomy & 15 & $18 \%$ \\
\hline Contraception & 02 & $02 \%$ \\
\hline Over Worked & 09 & $10 \%$ \\
\hline Food Induced & 14 & $17 \%$ \\
\hline Previous Delivery or Abortion & 08 & $09 \%$ \\
\hline Medicines & 02 & $02 \%$ \\
\hline No Known Cause & 08 & $09 \%$ \\
\hline
\end{tabular}

\section{Discussion}

Prevalence rate of excessive vaginal discharge was $24 \%$ in this study, implies that every fourth women attending gynecology out-patient department came with complain of excessive vaginal discharge. Similarly studies done by 
ChaudharyV et al., SinghAJ et al., Kulkarni RN et al. and Guntoory et al. reported $24.6 \%, 29 \%, 27.4 \%$ and $28.99 \%$ prevalence rate respectively ${ }^{1,7,8,11}$ while in community based survey done by BangRA et al. showed $13.5 \%$ rate. $^{12}$

Out of 720 women suffering from excessive vaginal discharge, no definite diagnosis can be made in 82 women, i.e., $11 \%$ women were included in study. As there was no biomedical cause of discharge identified, hence correlation between their socio-demographic and excessive vaginal discharge was tried to be established.

In study done by DeepaLM et al. specific diagnosis was obtained in $89 \%$ cases only. ${ }^{13}$ SivaranjaniR had found infectious cause in $51.7 \%$ cases, physiological discharge in $18 \%$ and no specific diagnosis made in $24.2 \%$ cases. ${ }^{14}$ In this study maximum cases were in age group 25-34 years $(59 \%)$ and next in group 35-44 years (30\%). In this age group, most women were over burdened by children, husband and family liabilities. Studies by Guntoory, PatelV and GeethaM reported higher prevalence rate in age group of 15-24 year while ChaudharyV showed high prevalence in age group above 40 years.

In this study more cases belonged to illiterate group (71\%), low socio-economic group (83\%) and women living in rural area (87\%). Guntoory, Kulkarni and Chaudhary also reported higher prevalence in low socio economic group. UmaD and JaspreetK reported higher prevalence in urban group may be due to high literacy and better facilities for reporting. ${ }^{15,16}$ PatelV reported higher prevalence in illiterate group. Women who worked as farmers (28\%), laborers $(33 \%)$, or who were home makers $(26 \%)$ suffered from excessive vaginal discharge more then who were skilled workers. Unhygienic surroundings, hard work and poor diet may be responsible. $70 \%$ women still goes for open air defecation although few women have toilets in their houses. Old habits do not die easily or scarcity of water may be the reason. $82 \%$ women were using cloths during periods and did not change them frequently. Most of women told that they can't afford to waste so much money on sanitary napkins. Similarly Guntoory and ManiG also noted a strong association between types of toilet and amount of vaginal discharge,.i.e., high prevalence in respondents who had no toilet facility at home. ${ }^{1,17}$ Guntoory found no association between discharge and use of sanitary pad while SinghAJ reported higher prevalence rate in women using rags as menstrual pads.

In study, higher prevalence was noted in gravida three or more and women who had home deliveries as compared to institutional deliveries. Chaudhary and Kulkarni found similar result in high parity group. Guntoory found no relation between parity and discharge but found high prevalence in home delivery cases. $65 \%$ women had undergone tubal sterilization in this study. Many women date back to tubal sterilization for their excessive vaginal discharge. May be tubectomy was done under compulsion or fear of some wrong happenings. Knowledge and interest in temporary methods of contraception is found to be very low. Not only women but whole family had unseen fears in use of temporary methods rather they prefer tubal sterilization after completion of family.

Nearly $83 \%$ women related multiple somatic symptoms to vaginal discharge. The most common perception that was held among these women was internal weakness. It was commonly believed that leucorrhoea makes body weak and in turn internal weakness increases the white discharge, so it is a to and fro process; it is an agent-cause-effect tirade. Most frequently reported symptom was leg cramps, backache, pain abdomen and bodyaches. Anxiety and stress arise due to these somatic symptoms and vice versa. Similar perceived effects were reported by Guntoory, KaurP, SinghAJ and Chaudhary. The logical link between discharge and other symptoms was almost impossible to establish.

In this study, perceived causes of leucorrhoea were multiple and mostly overlapped. The main factors that emerged to be causatives agent for excessive discharge included consumption of hot foods, internal weakness, allopathic medicine, body heat, economic hardship, hard work, fever, tubectomy and past bad delivery or abortion. Similar perceived causes of excessive discharge were noted by KaurP, SinghAJ, ChaudharyV.

The uniformity of associated symptom and perceived causes of excessive vaginal discharge raises the doubt whether these manifestation are real or psychological. Frustration and hopelessness were visible in women after months of treatment without any definite organic diagnosis. Psychologically upset to a certain degree always overpowered the main complaint. Hence vicious cycle is created with fear of life threating illness. Leucorrhoea may be both a discourse of distress or resistance, as well as a way of communicating about social and sexual concerns.

\section{Conclusion}

The study concludes that all vaginal discharge need not have any pathological or infectious basis hence biomedical diagnosis may not be possible in these cases. To avoid unnecessary lots of antibiotics, placebo treatments and unnecessary major surgical interventions, demographic status of these women should also be taken in consideration. Factors like age, illiteracy, low socio-economic status, poor personal and menstrual hygiene, high parity and place of delivery may not be causative but definitely contributory for occurrence of excessive normal vaginal discharge. So one of the most important part in management of these cases is proper counseling of women as well as of husband and family too. OommanNM et al. observed that poverty is the root cause which makes the women feel physiological weak. $^{18}$

So to bring positive change in ameliorating the problem of excessive vaginal discharge, improvement in education, socioeconomic status of women, increasing awareness about personal and menstrual hygiene, small family norms and advantages of use of temporary methods of contraception is essential. Health education programs to educate women about variation in amount of normal vaginal discharge should be encouraged through different media. Social and economic empowerments of women are the key areas of 
intervention to improve the socio-demographic status of women.

\section{Declaration}

This research paper is approved by ethical committee of AIIMS, Udaipur - 313001, Rajasthan.

\section{Conflict of Interest: None.}

Funding: None.

\section{References}

1. Guntoory I, Narasinga R, lakshmana R, Alina S. Prevelence and sociodemographic correlates of vaginal discharge among married women of reproductive age group at a teaching hospital. Int J Reprod Contracept Obstet Gynecol 2017;6(11):4840-6

2. Llewellyn-Jones,Funadamentals of obstetrics and gynecology. $9^{\text {th }}$ ed.2011, Mosby Elsevier limited

3. DuttaDc,Text Book of Gynaecology 6th ed.2013 Jaypee Brothers Medical publishers(P) Ltd, New Delhi, India

4. Kumar Jeffcoate's Principles of Gynaecology $8^{\text {th }}$ ed. New Delhi: Jaypee brothers Medical Publishers 2014.

5. Howkins and Bourne Show's Text Book of Gynaecology. $12^{\text {th }}$ ed. 2002 New Delhi: B. I. Churchill Livingstone.

6. Vishwanath S, Talwar V, Prasad R, Coyaji K, Elias CJ, de Zoysa I Syndromic management of vaginal discharge among women in a reproductive health clinic in India. Sex Transm Infect 2000;76(4):303-6.

7. Hawkes S, Morison L. foster S, Gausia K, Chakraborthy J, Weeling $\mathrm{R}$ et al Reproductive tract infections in women in in low income, low prevalence situations: assessment of syndromic management in Matlab, Bagladesh. Lancet 1999;354:1776-81.

8. Chaudhary V, Kumar R, Agrawal VK, Singh A, NarulaRm Sharma M. Prevelence and determinants of vaginal discharge among women of reproductive age group in Tertiary Care Hospital of Northern India. Natl J Community Med 2012;3(4):661-5.
9. Patel V, Pednekar S, Weiss H, Rodrigues M, Borros P, Nayak B et al.why do women complain of vaginal discharge ? A population survey of infectious and psychosocial risk factors in a south Asian Community. Int J Epidemiol 2005;34(4):853-62.

10. Singh AJ, vaginal discharge: Its causes and associated symptomsas perceived by rural north Indian women. Indian $J$ Comm Med 2007

11. Kulkarni RN, Durge PM, A study of leucorrhea in reproductive age group women of Nagpur city. Indian J Public Health 2005;49(4):238-9.

12. Bang R, Bang A, Women's perceptions of white vaginal discharge, ethnographic data from rural Maharasthtra. HarAnand Publication;1994:109-24.

13. Masand DL, Patel J, Gupta S. Utility of microbiological profile of symptomatic vaginal discharge in rural women of reproductive age group. J Clin Diagn Res 2015;9(3):QC04QC07.

14. Sivaranjini R, Jaisankar T, Thappa DM, Kumari R, Chandrasekhar L, Malathi M et al. Spectrum of vaginal discharge in a tertiary care setting. Trop Parasitol 2013;(2)135-9.

15. Uma D. A study of prevalence of leucorrhoea in women attending in OPD of obgyndeptt in a tertiary hospital. Int $J$ Res Health Sci 2013;1(3):230-4

16. Jaspreet K, Kapoor AK. Leucorrhea perception in a South Asian community. J Family Reprod Health 2014;8(1):45-52.

17. Mani G Prevalence of reproductive tract infections among rural married women in Tamil Nadu, India:A community based study. J Pioneer Med Sci 2014;4(1):18-24.

18. Oomann NM. Poverty and Pathology: comparing rural Rajasthani woman's ethnomedical models with biomedical models of reproductive morbidity: implications for womens health in India. Ann Arbor, Michigan, UMI Dissertation services. 1996:332(UMI No9629486).

How to cite this article: Ahmed SB. A Study on role of socio demographic status of women in excessive vaginal discharge at teaching hospital. Indian J Obstet Gynecol Res 2019;6(2):211-215 